\title{
MICROLEAKAGE EVALUATION OF ADHESIVE RESTORATIONS FOLLOWING CARIES REMOVAL USING CERAMIC BUR IN PRIMARY TEETH (AN IN VITRO STUDY)
}

\author{
Dalia M. Fathy ${ }^{1 * B D s}$, Magda M. El-Tekeya ${ }^{2} P h D$, Nevin S. Bakry ${ }^{2} P h D$, Mona H. Mohy El Din ${ }^{3} P h D$.
}

\begin{abstract}
INTRODUCTION: Conservative caries removal is the corner stone of minimally invasive dentistry. Ceramic bur is a self-limiting technique for caries removal in pediatric dentistry.

OBJECTIVES: The aim of the study is to evaluate microleakage of adhesive restorations (in vitro) using Ceramic bur to remove caries versus conventional drilling method.

MATERIALS AND METHODS: forty exfoliated carious primary teeth were allocated at random into two groups according to method of caries removal.

Group I ( $\mathrm{n}=20)$ caries removal by Ceramic bur, Group II ( $\mathrm{n}=20)$ caries removal by conventional tungsten carbide bur, followed by composite restorations of all prepared cavities.

Teeth were thermo-cycled for 500 cycles between $5^{\circ} \mathrm{C}$ and $55^{\circ} \mathrm{C}$, dwell time 10 seconds for 500 cycles and immersed into $1 \%$ methylene blue dye for $24 h$.

Teeth were sectioned in a bucco-lingual direction longitudinally and then studied using stereomicroscope at x20 magnification for dye penetration assessment. Data were collected and subjected to Mann Whitney U test.

RESULTS: No significant difference was noted in median microleakage scores and distance of stain penetration in millimeters between the two groups (Ceramic bur) and (conventional tungsten carbide bur) at a p value (0.097) and (0.289) respectively.

CONCLUSION: Microleakage of adhesive restorations is not adversely affected by caries removal using Ceramic bur.

KEYWORDS: Minimally invasive, ceramic bur, microleakage, primary teeth.

RUNNING TITLE: Ceramic bur effect on microleakage of adhesive restorations.
\end{abstract}

1. Bachelor of Dentistry, Faculty of Dentistry, Alexandria, Egypt.

2. Professor of Pediatric Dentistry, Department of Pediatric Dentistry and Dental Public Health, Faculty of Dentistry, Alexandria University, Alexandria, Egypt.

3. Professor of Dental Biomaterials, Department of Dental Biomaterials, Faculty of Dentistry, Alexandria University, Alexandria, Egypt.

* Corresponding Author:

E-mail: Dalia141289@gmail.com

\section{INTRODUCTION}

One of the widespread childhood diseases is dental caries. It is initiated as mineral loss and acid demineralization of enamel and dentin followed by degeneration of organic component leading to cavity formation $(1,2)$. It is a multifactorial disease disrupting the natural balance of remineralization- demineralization physiological process of tooth structure (3).

Histologically, Carious dentin is divided into; (a) superficial infected layer characterized by bacterial load. It is unrepairable and clinically detected as yellowish -brown soft layer, (b) Inner affected dentin layer is free from bacteria, reparable. It acquires a lighter color with hard leathery texture (4).

Conventional rotary method for caries removal enhanced the speed and efficiency of cavity preparation. However, this is associated with many drawbacks such as unpleasant vibration, frequent necessity of local anesthesia, harmful thermal, and pressure stimuli on the pulp resulting in an infected and affected carious dentin are removed aggressively (5).
The Minimally invasive approach combines preservation of tooth structure, remineralization, minimal intervention for carious tissue removal and placement of restorations, thus reaching the treatment goal with a less aggressive approach with minimal removal of healthy tissues to preserve tooth structure having the potential to remineralize. The widespread of this concept suggests that infected, heavily loaded with bacteria and permanently de-natured dentine is selectively removed to preserve sound re-mineralizable tooth structure (6).

Several techniques have been presented for caries removal as an alternative to conventional drilling include, air abrasion, sonoabrasion, and chemo-mechanical caries removal and selflimiting caries excavation burs; They have been shown to be promising methods in pediatric dentistry $(5,7)$.

The Ceramic bur (CeraBur K1SM, Komet-Brasseler, Lemego, Germany) has been developed with self -limiting technology for conservative cavity preparation. It is a round ended bur made of alumina-ceramic based material designed for slow speed caries excavation with minimal reduction of 
tooth structure $(7,8)$. It cuts easily through soft carious dentin and hardly through hard healthy dentin. Several studies evaluated its effectiveness on permanent teeth and found promising results $(5,7,9)$

A laboratory micro-CT investigation conducted in the year 2011 by Neves et al (7) compared the ceramic burs with eight minimally-invasive caries-removal techniques on primary teeth. The ceramic bur proved to have the best minimal invasive potential in terms of more conservative cavity preparation.

Clinical effectiveness of the Ceramic bur was studied in the year 2019 by El-Gheriany (8). It was concluded that the ceramic bur was as effective as the conventional tungsten-carbide bur in caries excavation of dentin of primary teeth. In addition, under SEM it produces a smooth dentin surface with partial elimination of the smear layer and opened dentinal tubules compared to tungsten carbide bur (8).

Marginal seal is one of the main factors affecting longevity of dental restorations. Additionally, proper attachment between the cavity walls and the restoration results in good marginal seal, less microleakage and a successful restoration (9).

Therefore, this study was intended to evaluate ceramic bur with a gold standard caries excavation method "tungsten carbide bur" to judge its effect on microleakage of adhesive restorations following caries removal in primary teeth.

The null hypothesis: is that no difference is expected in marginal seal of adhesive restorations between caries removal using Ceramic bur and conventional tungsten carbide bur.

\section{MATERIALS AND METHOD}

This study was done after approval of research ethic committee (0014-03/2019) in Faculty of Dentistry, Alexandria University.

Study design: Experimental in vitro study.

Setting and location: This study was performed at the Pediatric Dentistry and Dental Public Health Department, and Dental Biomaterials Department, Faculty of Dentistry, Alexandria University.

Sample size estimation: It was calculated using the values reported in a previous study by Wahba et al., in 2015 (11). They concluded that caries removal using smart bur II was less efficacious than conventional drilling using carbide bur regarding complete caries removal and that it required longer excavation times. However, conventional carbide bur removed more healthy dentine. Based on power of $80 \%$ and at a significance level of 95\%, 20 teeth in each group was needed, with total sample size $(n=40$ teeth) to generate a statistically significant result $(12,13)$.

Study sample: forty human Primary molars exfoliated or extracted for orthodontic purposes with Class I carious open cavities having medium to soft consistency and accessible to hand instruments were included in the study. Teeth with restorations, cracks, enamel defects or developmental anomalies were excluded. Teeth were washed and kept in distilled water. Teeth were collected from the out-patient clinics of the Oral Surgery Department and Orthodontic Department at Faculty of Dentistry, Alexandria University and Ministry of Health Hospitals, Alexandria.
Allocation and Randomization Teeth were randomly allocated based on caries removal method into two groups using random allocation software program. $(n=20)$.

Study outcome Microleakage evaluation through dye penetration scores as well as distance of stain penetration in millimeters.

Microleakage test Procedure: Teeth were sealed apically with sticky wax and embedded in chemically cured acrylic resin.

Group I Ceramic bur: caries was removed with Ceramic bur (CERA Bur K1SM, Komet-Brasseler, Lemego, Germany.) ISO size 014 and 016 according to the extent of the carious lesion, mounted on low-speed handpiece at $1500 \mathrm{rpm}$ approximately. Excavation endpoint was defined according to the operator's tactile sensation, where the bur cuts hardly when touching healthy hard dentin according to the manufacturer instructions (14).

Group II conventional tungsten carbide bur: caries was removed using tungsten carbide bur (Tungsten carbide round bur H1SE, Komet-Brasseler, Lemego, Germany) (10) of ISO size 014 and 016 according to the size of the carious lesion, mounted on low-speed handpiece at $1500 \mathrm{rpm}$ approximately. Caries excavation end point was when no discoloration was observed in remaining dentin visually, under proper light and by tactile sensation using an explorer $(10,15)$.

For all groups teeth were washed and dried, Self-etch (Sçotchbond $^{\mathrm{TM}}$ universal adhesive 3M ESPE, St. Paul, MN, USA) was applied in twenty seconds then air dried for five seconds and light cured for ten seconds, The cavities were restored with composite restoration (FilteK ${ }^{\mathrm{TM}} \mathrm{z} 250$ universal restorative 3M ESPE, St. Paul, MN, USA) and light cured for forty seconds, All steps were done according to manufacturer's instructions $(16,17)$.

Thermocycling: was done for 500 cycles between $5^{\circ} \mathrm{C}$ and $55^{\circ} \mathrm{C}$, dwell time 10 seconds for 500 cycles.

Dye penetration evaluation: After thermocycling, each tooth was painted with a nail polish leaving one mm short of the restorative margins. Then the samples were immersed in one percent methylene blue for $24 \mathrm{~h}$. Subsequently, samples were rinsed with pumice to remove the superficial dye (18). Degree of dye penetration was assessed under a stereomicroscope (Olympus SZ1145, Optical Co., LTD. Tokyo, Japan) at x20 magnification, the image appears on computer screen (Toup view, version 3.7, 2018).

- Microleakage score was recorded for each tooth. Each tooth produced two sections, both were examined, and the higher score was documented according to the criteria suggested in the year 2003 by Prabhakar A et al., (19) (Table 1).

Table (1): Showing dye penetration scores.

\begin{tabular}{|c|l|}
\hline Score & \multicolumn{1}{|c|}{ Criteria } \\
\hline 0 & No dye Penetration \\
\hline 1 & dye penetration limited to the enamel \\
\hline 2 & dye penetration past the enamel up to the dentin \\
\hline 3 & dye penetration reaching the pulp \\
\hline
\end{tabular}


- Microleakage was measured quantitatively and the distance of stain penetration was recorded in $\mathrm{mm}$, parallel to the long axis of the tooth (20).

\section{Statistical analysis}

Microleakage scores and distance of stain penetration was assessed using Mann Whitney $U$ test ( $P$ value $\leq 0.05)$. Data analysis was performed using IBM SPSS Statistics for Windows, version 23 (IBM Corp., Armonk, N.Y., USA).

\section{RESULTS}

\section{Microleakage evaluation}

For Group I (Ceramic bur): one specimen (5\%) with score 0 denoting no microleakage, 7 (35\%) specimens with score 1, 9 specimens (45\%) with score 2 and 3 specimens (15\%) with score 3.

For Group II (conventional tungsten carbide bur), 13(15\%) specimens showed score 1,12 (60\%) specimens showed score 2 and 5(25\%) specimens showed score 3. Using Mann Whitney U test, the median microleakage scores between the groups showed no significant difference ( $\mathrm{p}=0.097$ ) (Table 2, Fig.1).

Table (2): Showing microleakage scores in group I, and group II.

\begin{tabular}{|c|c|c||}
\hline $\begin{array}{c}\text { Microleakage } \\
\text { scores }\end{array}$ & $\begin{array}{c}\text { Group I Ceramic } \\
\text { bur } \\
(\mathbf{n = 2 0})\end{array}$ & $\begin{array}{c}\text { Group II Tungsten bur } \\
\text { (n=20) }\end{array}$ \\
\hline Score 0 & $1(5 \%)$ & $0(0 \%)$ \\
\hline Score 1 & $7(35 \%)$ & $3(15 \%)$ \\
\hline Score 2 & $9(45 \%)$ & $12(60 \%)$ \\
\hline Score 3 & $3(15 \%)$ & $5(25 \%)$ \\
\hline Median (IQR) & $2(1-2)$ & $2(2-2.75)$ \\
\hline Min - Max & $0-3$ & $1-3$ \\
\hline$P$-value & \multicolumn{2}{|c|}{0.097} \\
\hline
\end{tabular}

*Statistically significant difference at $\mathrm{p}$ value $\leq 0.05$
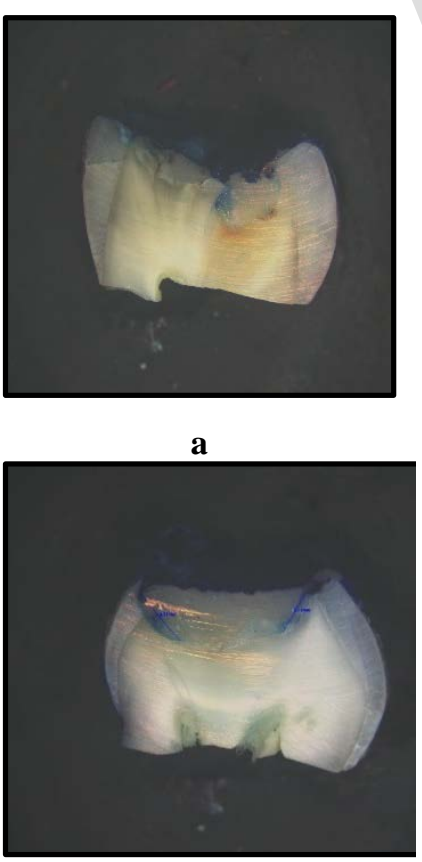

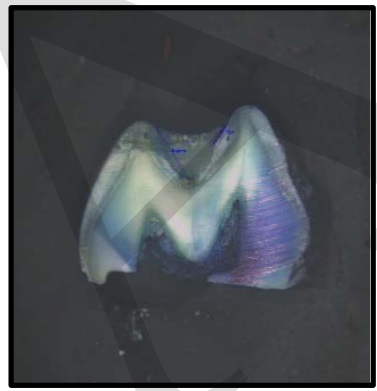

b

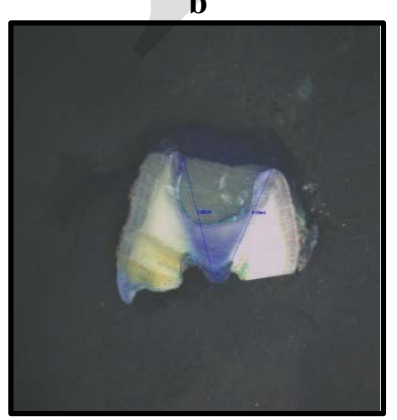

d
Figure (1): Showing microleakage in group I (a): score 0, (b): score 1, (c): score 2, (d): score 3.
Median distance of stain penetration for Group I (Ceramic bur) was (0.27) while for Group II (conventional tungsten carbide bur) was (0.29). No significant difference was noted in the median distance of stain penetration among the groups $(\mathrm{p}=0.289)$ (Table 3).

Table (3): Showing Distance of stain penetration in millimeters between Group I and Group II.

\begin{tabular}{||c|c|c||}
\hline $\begin{array}{c}\text { Microleakage } \\
\text { Distance of stain } \\
\text { penetration }\end{array}$ & $\begin{array}{c}\text { Group I } \\
\text { Ceramic bur } \\
(\mathbf{n = 2 0 )}\end{array}$ & $\begin{array}{c}\text { Group II } \\
\text { Tungsten bur } \\
\mathbf{( n = 2 0 )}\end{array}$ \\
\hline Median (IQR) & $0.27(0.18-0.46)$ & $0.29(0.22-0.45)$ \\
\hline Min - Max & $0-0.51$ & $0.11-0.68$ \\
\hline$P$-value & \multicolumn{2}{|c||}{0.289} \\
\hline
\end{tabular}

*Statistically significant difference at $p$ value $\leq 0.05$

\section{DISCUSSION}

The purpose of Minimally invasive dentistry is to maintain as much healthy enamel and dentin as possible during carious tissue removal. Carious dentin is composed of two layersinfected dentine and affected dentine. Affected dentin is capable of remineralization since the damage can be reversed, quality of bonding to dentin and marginal seal may be affected by the thickness of excavated dentin (9).

According to the manufacturer, Ceramic bur is highly efficient in selectively removing caries as it replaces both the excavator and explorer by simultaneously providing tactile sensation that is supposed to reduce the preparation time, the bur cuts through soft decayed dentin with absolute ease while hardly touching healthy hard dentin (7).

In the current study, the ceramic bur used was of the exact design of round bur as the tungsten carbide bur to eliminate any variables related to the bur design; consequently any results obtained are attributed solely to the material of which the bur is made. For each cavity, a brand-new bur was used to assure more standardization between cavities as regarding the sharpness of the bur.

The purpose of this study was to evaluate (in-vitro) the microleakage of adhesive composite restoration in class I cavities prepared on primary molars using Ceramic bur compared to conventional method, as the durability of the restoration is determined by marginal sealing of the cavity.

Randomization of the study sample according to caries removal method was done to make sure that all factors influencing the study outcome were equal, thus any detected difference in results could be accredited to difference in the tested material.

Teeth with enamel cracks or defects were eliminated from the study as they may affect the outcome of the study. To prevent dryness, teeth were cleaned and stored in distilled water soon after extraction to prevent them from dehydration until use. Distilled Water is the most favored storage solution, which results in minimal changes to enamel structure preserving organic and inorganic contents (21).

The caries excavation end point for the ceramic bur was defined by the tactile sensation of the operator when the bur hardly touches healthy hard dentin as it removes infected dentine only leaving affected dentine indicating self -limiting 
property of the ceramic bur, while tungsten carbide bur end point was no dentin discoloration as noted visually under adequate light as well as by tactile sensation using an explorer $(14,15)$

As Restoration success mainly depends on good marginal seal to reduce marginal deterioration, leakage, postoperative sensitivity, and secondary caries. Therefore, Microleakage would provide a better evaluation of different caries excavation techniques (9).

Laboratory assessment of the sealing potential of adhesive materials can be performed using organic dyes as tracers for microleakage. One of the most common methods for detecting defective adaptation of materials to tooth substrate such as (methylene blue, silver nitrate, basic fucsin). In the current study, samples immersed in 1\% methylene-blue dye for 24 hours. Methylene blue was applied in this study because it is easily applied, of low cost, and of low molecular weight (smaller than bacteria). Therefore, identifying leakage where bacteria could not penetrate (22-24).

Thermocycling was used to evaluate thermal aging under in vitro conditions. Controlled thermal changes in laboratoryenvironment have been used to simulate oral environment and to imitate the normal aging process in 6 months period $(25,26)$.

In the present study, Group I (Ceramic bur) showed less microleakage scores than group II (conventional tungsten carbide bur), there was no statistically significant difference (table 2, $\mathrm{P}=0.097$ ).

Regarding Median distance of stain penetration as a quantitative continuous parametric variable shown in table 3 , Group I (Ceramic bur) had a median of (0.27) which was less than that of group II (conventional tungsten carbide bur) (0.29) there was no statistically significant difference. (table $3, \mathrm{P}=0.289$ ).

This finding indicates that ceramic bur does not affect the sealing ability of adhesive restoration when compared with conventional tungsten carbide bur.

The mode of caries removal may affect the bond quality and adhesion of restorative material to the tooth structure to a considerable extent. In the year 2014 Pavuluri et al(18) stated that various techniques of caries removal result in different surface textures of excavated dentin and thickness of smear layer which can affect the quality of bonding to dentin and marginal seal $(19,20)$. In the year 2011 El Kassas et al (5) demonstrated that caries excavation with carbide bur led to a relatively compact smear layer while the ceramic bur produced a less compact and more porous smear layer. These findings were confirmed by El-Gheriany in 2019 (8) where he observed evident openings of dentinal tubules with few traces of smear layer following excavation with ceramic bur, whereas, following excavation by conventional tungsten carbide bur dentinal tubule openings were masked by smear layer deposits.

In light of the results of the present study, Ceramic bur as a minimally invasive mean for caries removal with self-limiting property in open dentinal lesions has promising outcomes. This is a consequence of good seal of composite restoration, which reinforce the results of previous clinical studies (8). Therefore, ceramic bur could be considered an effective reliable minimally invasive method for caries in primary teeth having the advantage of conserving tooth structure and providing residual caries-excavated dentin surface with higher bond strength, therefore greater bonding for adhesive restorative materials better than conventionally treated dentin (27).

A limitation of the present study was the lack of controlled environment of the natural oral condition; thermocycling did not entirely simulate the complex oral environment. Also, comparing ceramic burs with other minimally invasive methods of caries excavation may possibly have showed a wider range of results.

Therefore, further in vivo studies comparing the ceramic bur with different methods of caries excavation are recommended with emphasis on restorative and adhesive characteristics. Moreover, in vivo studies with special attention to the influence of the use of the ceramic bur on child behavior and pain perception are also needed.

The null hypothesis: was accepted as there was no statistically significant difference between the microleakage scores and distance of stain penetration of adhesive restorations following caries removal by Group I (Ceramic bur) and Group II (Conventional Tungsten carbide bur).

\section{CONCLUSION}

Microleakage of composite restorations is not adversely affected by caries removal using Ceramic bur.

\section{CONFLICT OF INTEREST}

The authors acknowledged that there was no conflict of interest.

The authors received no funding for their work.

\section{REFERENCES}

1. Featherstone JD. The science and practice of caries prevention. J Am Dent Assoc. 2000; 131:887-99.

2. Featherstone JD. The continuum of dental caries-evidence for a dynamic disease process. J Dent Res. 2004; 83:C39-42.

3. Featherstone JD. Caries prevention and reversal based on the caries balance. Pediatr Dent. 2006; 28:128-32.

4. Banerjee A, Watson TF. Pickards Guide to Minimally Invasive Operative Dentistry: OUP Oxford; 2015.

5. Elkassas DW, Elhoshy AZ, Abdalla AI. Effect of caries removal using ceramic bur on the microtensile bond strength of etch and rinse and self-etch adhesive systems. Int J Clin Dent. 2011; 4:11-20.

6. de Almeida Neves A, Coutinho E, Cardoso MV, Lambrechts P, Van Meerbeek B. Current concepts and techniques for caries excavation and adhesion to residual dentin. J Adhes Dent. 2011; 13:7-22.

7. Neves Ade A, Coutinho E, De Munck J, Van Meerbeek B. Caries-removal effectiveness, and minimal-invasiveness potential of caries-excavation techniques: a micro-CT investigation. J Dent. 2011; 39:154-62.

8. El-Gheriany. Evaluation of dentin caries excavation using Ceramic bur in primary teeth. M.Sc. Thesis. Pediatric Dentistry and Dental Public Health Department, Faculty of Dentistry, Alexandria University. Egypt. 2019.

9. Dammaschke T, Vesnic A, Schafer E. In vitro comparison of ceramic burs and conventional tungsten carbide bud 
burs in dentin caries excavation. Quintessence Int. 2008; 39:495-9.

10. Hafez MA, Elkateb M, El Shabrawy S, Mahmoud A, El Meligy O. Microleakage Evaluation of Composite Restorations Following Papain-Based Chemo-Mechanical Caries Removal in Primary Teeth. J Clin Pediatr Dent. 2017; 41:53-61.

11. Wahba W, Sharaf A, Bakery N, Nagui D. Evaluation of polymer bur for carious dentin removal in primary teeth. Alex Dent J. 2015; 40:107-12.

12. Liao JJ. Sample size calculation for an agreement study. Pharm Stat. 2010; 9:125-32.

13. Pannucci CJ, Wilkins EG. Identifying and avoiding bias in research. Plast Reconstr Surg. 2010; 126:619-25.

14. CERA line user manual. Available at:

http://cms.kometdental.de/fileadmin/_migrated/media/41009 5V1_BRO_EN_Ceraline.pdf

15. Munshi AK, Hegde AM, Shetty PK. Clinical evaluation of Carisolv in the chemico-mechanical removal of carious dentin. J Clin Pediatr Dent. 2001; 26:49-54.

16.3m ESPE, USA. Instructions for use of Scotchbond ${ }^{\mathrm{TM}}$ universal adhesive. Available at: https://multimedia.3m.com/mws/media/7937060/3mscotchbond-universal-adhesive-general-application.pdf

17.3m ESPE, USA. Instructions for use of Filtek z250 universal

restorative. Available at: https://multimedia.3m.com/mws /media/1821930/3m-filtek-z250-universal-restorativedirect-posterior-restorations.pdf

18. Pavuluri C, Nuvvula S, Kamatham RL, Nirmala SV. Comparative evaluation of microleakage in conventional and RMGIC restorations following conventional and chemomechanical caries removal: An in vitro study. Int J Clin Pediatr Dent. 2014; 7:172.

19. Prabhakar A, Madan M, Raju O. The marginal seal of a flowable composite, an injectable resin modified glass ionomer and a compomer in primary molars--an in vitro study. J Indian Soc Pedod Prev Dent. 2003;21:45-8.
20. Bonilla ED, Stevenson RG, Caputo AA, White SN. Microleakage resistance of minimally invasive Class I flowable composite restorations. Oper Dent. 2012; 37:290-8.

21. de Melo Maranhão K, Klautau EB, Pereira Pd, Guimarães RB, Pantoja VG. The effect of storage solutions on enamel of bovine teeth. Salusvita, Bauru. 2009; 28:129-34.

22. Hegde S, Kakti A, Bolar DR, Bhaskar SA. Clinical Efficiency of Three Caries Removal Systems: Rotary Excavation, Carisolv, and Papacarie. J Dent Child (Chic). 2016;83:22-8.

23. Ertürk Avunduk AT, Bağlar S. Evaluation of microleakage in class $\mathrm{v}$ cavities prepared by different caries removal methods. Microsc Res Tech. 2019;82:1566-74.

24. Patel MU, Punia SK, Bhat S, Singh G, Bhargava R, Goyal $\mathrm{P}$, et al. An in vitro evaluation of microleakage of posterior teeth restored with amalgam, composite and zirconomerA stereomicroscopic study. J Clin Diagn Res. 2015;9:ZC65-7.

25. Korkmaz Y, Gurgan S, Firat E, Nathanson D. Effect of adhesives and thermocycling on the shear bond strength of a nano-composite to coronal and root dentin. Oper Dent. 2010;35:522-9.

26. The ISO TR 11450 standard. International Organization for Standardization. ISO TR 11450. Dental materialsguidance on testing of adhesion to tooth structure. 1994.

27. Neves Ade A, Coutinho E, Cardoso MV, de Munck J, Van Meerbeek B. Micro-tensile bond strength and interfacial characterization of an adhesive bonded to dentin prepared by contemporary caries-excavation techniques. Dent Mater. 2011;27:552-62. 